\title{
Mechanotherapeutics for the Treatment of Idiopathic Pulmonary Fibrosis
}

\author{
Hongwei Han, $\mathrm{PhD}^{1,2,3}$ and David Lagares, $\mathrm{PhD}^{1,2,3}$
}

${ }^{1}$ Department of Medicine, Division of Pulmonary and Critical Care Medicine; ${ }^{2}$ Fibrosis Research Center; ${ }^{3}$ Center for Immunology and Inflammatory Diseases, Division of Rheumatology, Allergy and Immunology. Massachusetts General Hospital, Harvard Medical School, Boston, USA

\section{ABSTRACT}

Idiopathic pulmonary fibrosis (IPF) is an age-related progressive lung disease characterized by excessive deposition of extracellular matrix (ECM) produced by activated myofibroblasts. Traditionally, myofibroblast activation has been thought to be exclusively driven by soluble biochemical stimuli, such as pro-fibrotic growth factors and cytokines. However, the mechanical properties of the fibrotic ECM including matrix stiffness have recently gained more attention given its ability to drive myofibroblast activation independently from soluble mediators. The study of fibroblast mechanobiology is an active area of research in IPF and focuses on understanding how matrix stiffness is sensed and translated into biochemical signaling via the so-called mechanotransduction pathways, which ultimately regulate profibrotic gene expression, ECM synthesis and myofibroblast survival. Here, we summarize the molecular mechanisms promoting mechano-activation of myofibroblasts in lung fibrosis and the potential of treating IPF with "mechanotherapeutics", a novel class of anti-fibrotic therapeutic agents. (BRN Rev. 2021;7(2):96-108)

Corresponding author: David Lagares, dlagares@mgh.harvard.edu

Key words: Apoptosis. Idiopathic pulmonary fibrosis. Matrix stiffness. Mechanotherapeutics. Myofibroblasts. 


\section{INTRODUCTION}

Lung fibrosis is defined as scarring in the lungs. It is generally induced by repeated or chronic injury to the alveolar epithelial cells, which leads to activation of inflammatory responses and subsequent fibrotic tissue repair ${ }^{1}$. Upon lung injury, pulmonary fibroblasts get activated and become extracellular matrix (ECM)-secreting myofibroblasts, which are responsible for restoring the normal tissue structure by secreting, contracting and remodeling of the $\mathrm{ECM}^{2}$. During the resolution of the normal tissue repair program, myofibroblasts are cleared out by immune cells after their mission is completed $^{3,4}$. However, they persist in the context of lung fibrosis, maintaining their activated state and promoting progressive scarring of the lungs ${ }^{5}$. Thus, myofibroblast persistence is a hallmark of lung fibrosis and their numbers have been shown to correlate with disease stage and progression in idiopathic pulmonary fibrosis (IPF) ${ }^{6}$, the most common and aggressive type of pulmonary fibrotic diseases. The continuous presence of activated myofibroblasts leads to ECM built up, which is also crosslinked and stiffened by matrix crosslinking enzymes. We and others have shown that ECM stiffness is dramatically increased during the development and progression of lung fibrosis ${ }^{7}$. Such alteration in the ECM mechanical properties is now recognized as a major driver of lung fibrogenesis by promoting mechano-activation of fibroblasts ${ }^{8,9}$. In this Review, we will discuss the mechanobiology of lung fibrosis and summarize novel anti-fibrotic strategies for the treatment of lung fibrosis with the so-called mechanotherapeutics.

\section{IPF}

IPF is a progressive, irreversible, and typically lethal lung fibrotic disease without any known cause. It is thought to be initiated by alveolar epithelium injury caused by a complex interplay of genetic factors and environmental insults, followed by inflammation, activation of myofibroblasts and excessive ECM deposition within the lung parenchy$\mathrm{ma}^{10}$. The damaged lung tissue becomes stiff and thick, ultimately limiting the amount of oxygen that gets into the blood. IPF is associated with high mortality, with a reported median survival of two-three years post-diagnosis, and the incidence of the disease is rising rapidly worldwide ${ }^{10}$, with doubling of prevalence between 2000 and $2012^{11}$.

Currently, there is no cure for IPF. In 2014, two novel medicines, pirfenidone and nintedanib, were approved for the treatment of IPF. These drugs modestly slow down the progression of IFP but do not halt or reverse $\mathrm{it}^{12,13}$. In addition, neither of the two medicines elevate patients' perceived quality of life, and both agents confer substantial side effects (nausea and rash with pirfenidone; diarrhea and abnormal liver function with nintedanib) ${ }^{14}$. Therefore, novel therapeutics are highly needed for the treatment of lung fibrosis in patients with IPF.

\section{MECHANOBIOLOGY OF LUNG FIBROSIS}

Over the last 20 years, the vast majority of therapeutic strategies aimed at treating lung fibrosis is focused on targeting biochemical factors including pro-fibrotic growth factors 
and cytokines ${ }^{15,16}$. More recently, biophysical factors such as mechanical forces and matrix stiffness have gained increasing recognition as critical regulators of lung fibrosis development and progression ${ }^{17,18}$. The mechanobiology of lung fibrosis is gaining increasing attention not only because it is revealing novel disease mechanisms but also by providing new therapeutic targets for the treatment of lung fibrosis. One of the hallmarks of lung fibrosis is the dramatic increase in matrix rigidity or stiffness, which is largely regulated by activated myofibroblasts ${ }^{9,19-21}$. Stiffness is defined as the resistance to deformation in response to applied force. It is typically measured by Young's elastic modulus (E), which is expressed as the tensile stress (force per unit area) divided by the strain (deformation). The unit is Pascals $\left(\mathrm{Pa}, \mathrm{N} / \mathrm{m}^{2}\right)$. In normal lung tissue, the tissue stiffness is usually maintained between 0.5 to $2 \mathrm{kPa}$. This is critical during tissue homeostasis since the ECM composition and rigidity provide a matrix scaffold that regulates normal physiological processes such as cell adhesion, proliferation and migration ${ }^{22,23}$. Fibroblasts constantly secrete, degrade and remodel the ECM in order to maintain the integrity of the matrix, both biochemically and biophysically ${ }^{24}$. They conduct mechanical measurements, a process known as mechanosensation, by actively pulling on their environment and evaluating whether mechanical homeostasis has been disrupted through specific positive and negative feedback mechanisms ${ }^{8}$. For instance, evidence has shown that ECM degradation leads to increased ECM synthesis and deposition by fibroblasts. On the other hand, matrix stiffness modulates expression of collagen-degrading enzymes such as MMP-1 in fibroblasts ${ }^{21,25,26}$. Besides ECM composition, fibroblasts further adjust matrix stiffness by secreting matrix crosslinking enzymes including lysyl oxidases (LOXs) and transglutaminases (TGs) ${ }^{27}$. Together, fibroblasts maintain the ECM stiffness within a normal range, which is necessary for normal physiological processes. However, such homeostatic feedback mechanism between fibroblasts and ECM is disrupted upon lung injury. In this context, fibroblasts are activated to myofibroblasts, a cellular phenotype characterized by increased ECM synthesis and contractility ${ }^{28,29}$. In the early stages following injury, myofibroblasts actively secrete ECM proteins to provide a tissue scaffold for normal repair events such as epithelial cell migration ${ }^{30}$. In later stages of tissue repair, myofibroblasts facilitate wound closure and re-epithelialization with their enhanced contractile abilities, which is conferred by upregulation of -smooth muscle actin (SMA) ${ }^{31,32}$. Of note, matrix stiffness is a major driver of SMA expression during fibroblast-to-myofibroblast transdifferentiation during wound healing $^{7,17,18}$. The increased ECM stiffness not only promotes fibroblast mechano-activation but also their survival. In this regard, decreased matrix stiffness during the resolution of the normal tissue repair program has been shown to induce myofibroblast apoptosis ${ }^{5}$. However, this mechanical checkpoint goes awry in fibrotic disorders, creating a vicious positive feedback loop between ECM stiffness and myofibroblasts that results in myofibroblast persistence, pathological ECM deposition and fibrosis ${ }^{33}$. In this amplification loop, myofibroblasts extensively secrete type I collagen and covalently cross-link the ECM via LOX and TG2, resulting in collagen fibers that are more resistant to degradation. This stabilized ECM leads to a dramatic increase in matrix stiffness, which can be up to $40 \mathrm{kPa}$ at the late stage of lung fibrosis $^{21,34,35}$. Moreover, matrix stiffness can also activate surrounding quiescent fibroblasts through 
mechano-transduction pathways, amplifying fibrotic responses. In summary, this mechanical positive feedback loop is a crucial driving force during the development and progression of lung fibrosis. In this Review, we describe the biological and molecular mechanisms behind this pathological mechanism in IPF and discuss novel therapeutic strategies to break this vicious positive feedback loop, which have been shown to ameliorate lung fibrosis in preclinical models.

\section{MECHANOTHERAPEUTICS FOR THE TREATMENT OF LUNG FIBROSIS}

\section{Targeting matrix crosslinking enzymes}

Two major families of matrix crosslinking enzymes, LOX and TG, have been shown to be upregulated in patients with $\mathrm{IPF}^{36,37}$. Members of the LOX family comprise LOX, and LOX-like LOXL1, LOXL2, LOXL3 and LOXL4. The central function of LOX enzymes is to catalyze the covalent crosslinking of collagen and other ECM proteins by oxidizing peptidyl lysine to form peptidyl $\alpha$-aminoadipic- $\delta$-semialdehyde. These aldehyde residues can spontaneously condense with neighboring peptidyl lysines or peptidyl aldehyde, leading to the formation of insoluble aggregates found in fibrillar collagen, thus stabilizing the ECM (Fig. 1). It has also been shown that the crosslinking of ECM enhances fibroblast proliferation and inhibits matrix degradation in lung fibrosis ${ }^{38}$. LOX family members are upregulated in fibrotic diseases, especially LOX/LOXL1/LOXL2 ${ }^{39,40}$. Pan-inhibition of LOX enzymes with the non-specific inhibitor (BAPN) treats lung fibrosis in mice $^{41}$. LOXL1 knockout mice are similarly protected from lung fibrosis in preclinical models ${ }^{42}$. Simtuzumab, a humanized monoclonal antibody against LOXL2, has been shown to treat lung fibrosis in mice and was recently tested in phase 2 trials to treat lung fibrosis in patients with definite $\mathrm{IPF}^{43}$. Unfortunately, simtuzumab did not improve progression-free survival in patients with IPF, and Gilead Sciences terminated its phase 2 clinical study due to lack of efficacy ${ }^{44}$. The failure of this trial is attributed to lack of tissue penetration by simtuzumab in human IPF lungs. Novel small molecules targeting LOXL2 are currently under investigation ${ }^{45,46}$. Among them, two selective small molecule LOXL2 inhibitors: PXS-5382A and PXS-5338K, developed by Pharmaxis, have been recently announced ready for phase 2 trials in patients with IPF and NASH (ACTRN12617001444370 and ACTRN12617001564347). Another panLOX Inhibitor (PXS-5505A) by the same pharmaceutical company is ready to enter phase 2 studies in patients with myelofibrosis (ACTRN12619000332123). TG2, also known as tissue transglutaminase, has been also shown to be highly upregulated during lung fibrosis ${ }^{37,47}$. In humans, TG2 expression and activity have been shown increased in lung tissue from patients with IPF compared with normal control individuals ${ }^{47,48}$. TG2 knockout mice are protected from lung fibrosis in mice and pharmacological TG2 inhibition similarly treats preclinical lung fibrosis ${ }^{37,47}$. Pharmacological inhibition of TG2 has been shown to treat bleomycin-induced pulmonary fibrosis in mice through inhibiting $\mathrm{EMT}^{49}$. Zedira recently announced that their ZED1227, a first-in-class tissue transglutaminase inhibitor, has shown good safety and tolerability and is undergoing phase $2 \mathrm{a}$, double-blind trails for Celiac disease (2017-002241-30); and 


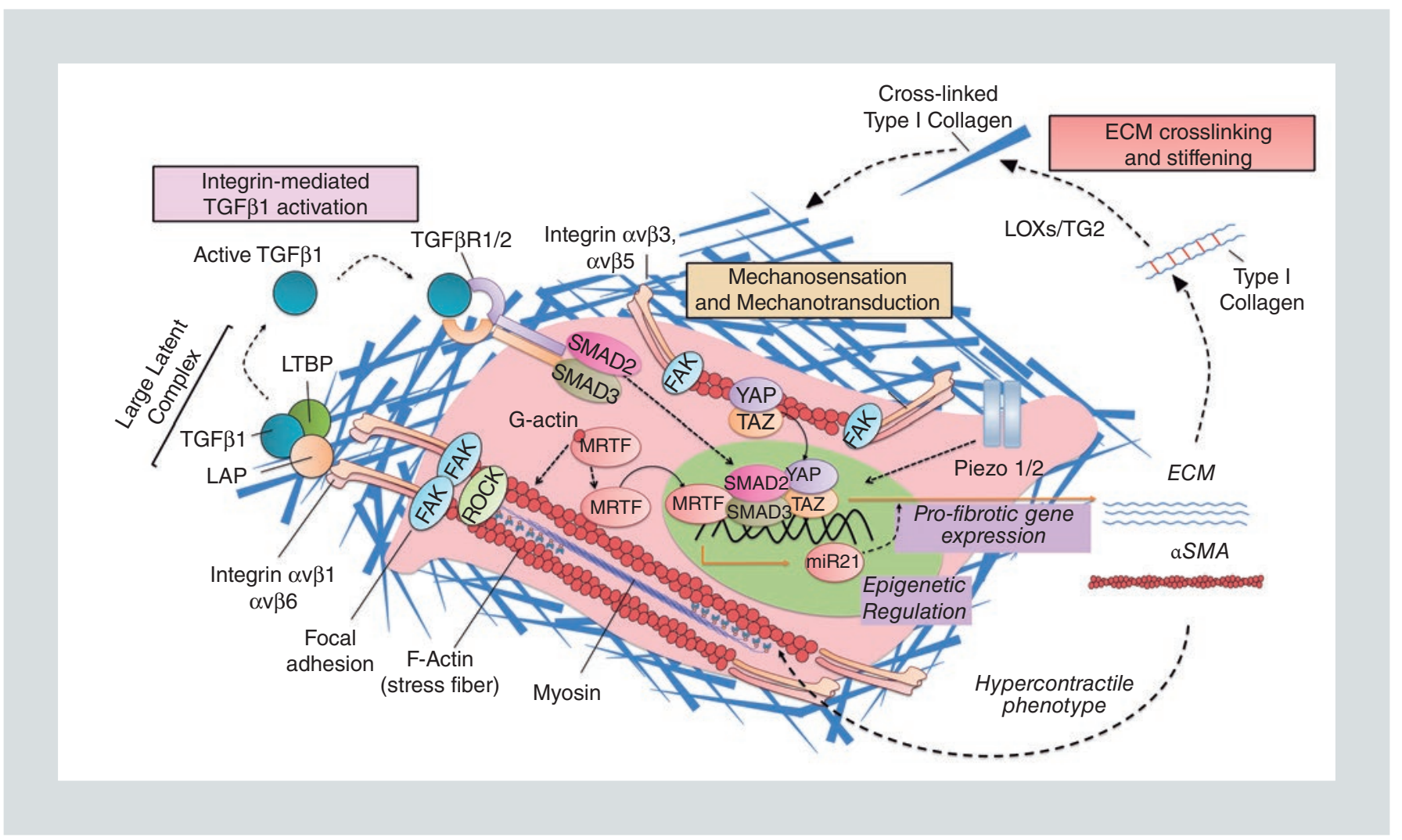

Figure 1. Mechanoactivation of fibroblasts by matrix stiffness. Matrix stiffness is increased dramatically during the development of lung fibrosis. Fibroblasts sense changes in matrix stiffness through mechanosensors on the cell membrane including integrins and mechanosensitive ion channels such as Piezo channels. Integrin-mediated mechanotransduction is a primary mechanism by which cells translate mechanical signals into biochemical signaling pathways. Mechanotransduction pathways activate downstream signaling including FAK, ROCK, MRTF-A/B, and YAP/TAZ, which drives the expression of profibrotic genes such as Type I collagen and $\alpha$ SMA. Type I collagen can be further stabilized by two major matrix crosslinking enzymes including lysyl oxidases (LOX) and transglutaminases (TG2), further increasing matrix stiffness. Once activated, myofibroblasts exert higher traction forces that promote mechano-activation of the potent profibrotic cytokine TGF- $\beta 1$ through integrins $\alpha v \beta 6$ and $\alpha v \beta 1$, amplifying the fibrotic response via TGF- $\beta$ receptor signaling. Together, mechanical forces generate a progressive positive feed-forward loop in which enhanced matrix deposition and tissue stiffening lead to fibroblast mechano-activation, which further secretes excessive ECM and promotes matrix stiffening (adapted from Tschumperlin DJ et al. ${ }^{7}$ with permission from Elsevier, (C) 2020 Elsevier Inc. All rights reserved).

ECM: extracellular matrix; FAK: focal adhesion kinase; IPF: idiopathic pulmonary fibrosis; LAP: latency-associated peptide; LOX: Iysyl oxidase; LTPB: latent TGF- $\beta 1$ binding proteins; miR: microRNA; MRTF: myocardin-related transcription factor; ROCK: rho-associated protein kinase; TAZ: transcriptional coactivator with PDZ-binding motif; TGF- $\beta 1$ : transforming growth factor $\beta 1$; YAP: yes-associated protein.

they plan to target organ fibrosis including lung, liver, and kidney fibrosis.

\section{Targeting fibroblast mechano-activation}

As described above, the stiffness of fibrotic lungs $(15-100 \mathrm{kPa})$ is much higher than the normal lung parenchyma $(0.5-2 \mathrm{kPa})^{9,50,51}$. We and others have shown that tissue stiffening in IPF contributes to the onset and progression of lung fibrosis by influencing fibroblast behavior and function, in particular by promoting fibroblast mechano-activation and survival ${ }^{18,21,28,52}$. The study of fibroblast mechanobiology is an active area of research in IPF and focuses on understanding how matrix stiffness is sensed 
and translated into biochemical signaling pathways, ultimately affecting profibrotic gene expression, ECM synthesis and survival. The molecular mechanisms beneath fibroblast mechanoactivation are not fully understood, but two successive molecular steps, mechanosensing and mechanotransduction, have been recognized as major regulators of fibroblast mechanobiology 7,53,54. Mechanosensing is a cellular process in which cells actively assess the stiffness of the matrix ${ }^{53,55}$. ECM receptors called integrins play a central role in cellular mechanosensing 56,57 . Two members of the integrin family, $\alpha v \beta 1$ and $\alpha v \beta 3$, have been shown to play central roles at transducing mechanical cues into biochemical signaling in fibroblasts. Recent studies have shown that $\alpha v \beta 3$ integrin regulates fibroblast contractility and matrix stiffening 58 , and that pharmacological inhibition of $\alpha v \beta 3$ integrin attenuates fibrosis in mice ${ }^{59}$. Similarly, $\alpha v \beta 1$ integrin has been shown to promote tissue fibrogenesis in vivo by integrating fibrogenic mechanical cues ${ }^{60}$. Accordingly, pharmacological and genetic inhibition of $\beta 1$ integrin have been reported to ameliorate fibrosis in mouse models ${ }^{60}$. Integrins physically link the actin cytoskeleton to the ECM through cell-matrix adhesion complexes termed "focal adhesions" (FAs) ${ }^{56}$ (Fig.1). Focal adhesions are membrane-associated multi-protein complexes that transmit cell-exerted forces to the matrix ${ }^{61}$. They are involved in multiple cellular processes such as cell survival, proliferation and motility ${ }^{62}$. Focal adhesions proteins include focal adhesion kinase (FAK), paxillin, vinculin and talin, which undergo mechanical activation in response to force-induced protein stretching $^{63}$. FAs dynamically sample matrix stiffness by applying pulling forces to the ECM through actomyosin contraction ${ }^{64}$. On soft matrices, cell-mediated traction forces are low and focal adhesion proteins remain intact. However, on stiff matrices as in pathological fibrosis, cell exerted traction forces are high, ultimately stretching and activating focal adhesion proteins ${ }^{56,65}$. Whereas the biology of focal adhesion proteins in fibroblast mechanotranduction remains to be fully elucidated, it is well established that FAK undergoes dynamic changes in response to force-induced protein stretching. Mechanical stretching of FAK leads to the activation of its catalytic domain and subsequent phosphorylation of downstream signaling proteins ${ }^{67}$, which is implicated in the initiation of mechanotransduction signaling ${ }^{67,68}$. We and others have shown that FAK is consistently activated in myofibroblasts during lung fibrosis ${ }^{67,69}$ and that pharmacological inhibition of FAK mitigates lung fibrosis in mice ${ }^{67}$. Defactinib, a FAK inhibitor developed by Verastem Oncology (VS-6063, PF-04554878), has entered phase 2 trials to investigate its anti-fibrotic effects in patients with pancreatic cancer (NCT02758587). Although FAK inhibitors have shown potent anti-fibrotic effects in preclinical models and are now being investigated in humans, fibrogenic signaling pathways activated by FAK in fibroblasts are not fully understood and remain actively investigated. Mechano-activated FAK drives myofibroblast activation via Rho/Rho-associated protein kinase (ROCK) mediated yes-associated protein (YAP)/transcriptional coactivator with PDZ-binding motif (TAZ) and myocardin-related transcription factor (MRTF) pathways ${ }^{70}$ and prevents myofibroblast apoptosis by upregulating the anti-apoptotic protein B-cell lymphoma-extra-large (BCL-XL) ${ }^{5,52}$. Rho/ ROCK pathway is a well-studied downstream signaling of $\mathrm{FAK}^{71,72}$. In vitro, pharmacological, and genetic inhibition of ROCK prevents myofibroblast differentiation induced by matrix stiffness and global 
haploinsufficient ROCK1 and ROCK2 mice are protected from lung fibrosis in bleomycin-induced mouse model ${ }^{71-73}$. Currently, ROCK inhibitors have not entered human clinical trials for the treatment of organ fibrosis although a panROCK inhibitor, Fasudil, has been approved for treatment of coronary and cerebral vasospasm in humans ${ }^{74}$. The use of pan-ROCK inhibitors for treating fibrosis is under debate due to its potential side effect of systemic hypotension ${ }^{75}$. The development of isoform-specific ROCK inhibitors might solve the safety issue. KD025 (also called SLX-2119), the first specific ROCK2 inhibitor, has been developed by the biopharmaceutical company Kadmon and is currently tested in phase 2 study (NCT03640481). Mechanotransduction pathways ultimately lead to activation of transcriptional factors and co-activators that drive pro-fibrotic gene expression. Two sets of transcriptional coactivators: YAP and TAZ and MRTF-A and MRTF-B, are recognized as major mechanotransducers ${ }^{76,77}$. A growing body of evidence has shown that the activity of the transcriptional coactivators YAP and TAZ, effector proteins of the Hippo pathway that shuttle from the cytoplasm to the nucleus to control gene expression, is regulated by matrix stiffness ${ }^{78}$. On soft matrices, the large tumor suppressor kinase 1/2 (LATS1/2) phosphorylates YAP/TAZ on residues S127 and S381, leading to sequestration of YAP/TAZ in the cytoplasm and subsequent degradation via the ubiquitin proteasome system ${ }^{79}$. However, under high mechanical loading, YAP/TAZ avoid cytoplasmic retention and degradation by yet unknown mechanisms, leading to their translocation to the nucleus ${ }^{80}$ where they bind to transcription factors including TEA domain (TEAD) and SMADs, ultimately promoting expression of pro-fibrotic genes such as connective tissue growth factor (CTGF), $\alpha$-SMA and type I collagen ${ }^{81-83}$ (Fig.1). Thus, mechanical YAP/TAZ signaling directly drives myofibroblast activation, proliferation, and ECM secretion. In addition, we have shown that YAP/TAZ signaling also promotes the expression of the pro-survival BCL-2 protein BCL-XL, thus promoting myofibroblast evasion of apoptosis ${ }^{52}$. In vivo, TAZ-heterozygous mice showed protection from lung fibrosis in bleomycin-induced lung fibrosis model ${ }^{82}$, and specific depletion of YAP/TAZ in fibroblasts displayed reduced kidney fibrosis in mice ${ }^{84,85}$. Multiple strategies targeting YAP/TAZ pathways are under investigation including selective inhibition of YAP/TAZ via dopamine receptor D1 agonist $^{86}$ and stimulation of YAP degradation through multiple hydroxymethylglutaryl-coenzyme A (HMG-CoA) reductase inhibitors (statins) $^{87}$. In addition to YAP/TAZ, both MRTF-A and MRTF-B have been also reported as critical mediators of mechanotransduction pathways. MRTF-A and MRTF-B are transcriptional coactivators linking actin dynamics to serum response factor (SRF)-mediated gene transcription ${ }^{88}$. MRTF-A and -B are predominantly localized to the cytoplasm and only translocate to the nucleus upon stimulation. Nuclear translocation of MRTF is controlled by Rho GTPases via actin dynamics stimulated by matrix stiffness ${ }^{89}$. MRTFs bind monomeric G-actin molecules via three N-terminal RPEL motifs, sequestering them in the cytoplasm $^{89}$. Rho-mediated actin polymerization and subsequent formation of filamentous F-actin releases MRTFs, resulting in increased nuclear accumulation where they bind SRF to drive transcription of pro-fibrotic genes such as $\alpha-\mathrm{SMA}^{90}$ (Fig.1). MRTF-A depletion in mice conferred protection from lung, cardiac and kidney fibrosis in preclinical models ${ }^{77,91-93}$, and that inhibition of MRTF-A/B with the small 
molecule inhibitor CCG-203971 diminished lung and skin fibrosis in mice ${ }^{93,94}$.

In addition to integrins, other mechanosensitive receptors have been shown to regulate fibroblast mechano-activation in the context of tissue fibrosis $^{33}$. For instance, the family of transient receptor potential channels (TRP) has been involved in fibroblast mechanosensing and tissue fibrogenesis ${ }^{95}$. TRP channel family majorly includes TRPA, TRPC, TRPM, TRPML and $\mathrm{TRPV}^{96}$, and they are involved in various types of sensory reception, including thermoreception, chemoreception, mechanoreception, and photoreception ${ }^{97-99}$. In particular, the mechanosensitive transient receptor potential vanilloid 4 channel (TRPV4) has been reported to mediate stiffness-activation of lung and cardiac fibroblasts in vitro $^{100,101}$. Accordingly, global TRPV4 knockout mice showed protected against bleomycin-induced lung fibrosis in mice ${ }^{100}$. Moreover, the calcium-activated Piezo1 and 2 channels have been similarly shown to be mechanically activated in human diseases ${ }^{102,103}$. Piezo1 has been reported to be the primary sensor of mechanical stress in suppressive myeloid cells and genetic ablation of Piezo1 in mice protects against cancer and polymicrobial sepsis by diminishing immunosuppressive activities of myeloid cells ${ }^{104}$. It has been recently shown that mechanically activated Piezo1 enhances the production of profibrotic cytokine interleukin-6 (IL-6) through activating p38 mitogen-activated protein kinase (MAPK) in cardiac fibroblasts ${ }^{105}$.

\section{Targeting mechanical control of TGF-ß1 activation}

TGF- $\beta 1$ is the most studied pro-fibrotic cytokine and recognized as the master regulator of fibrosis due to its ability to drive tissue fibrosis in vivo in multiple organs ${ }^{106}$. It has been shown that TGF- $\beta 1$ promotes myofibroblasts activation, resistance to apoptosis and ECM synthesis ${ }^{107}$. TGF- $\beta 1$ is a very pleiotropic cytokine involved in immune suppression and immunotolerance ${ }^{108}$, therefore its activity and availability must be tightly regulated. TGF- $\beta 1$ is secreted as a latent cytokine and stored in the ECM. During its synthesis and modification in the Golgi, mature TGF- $\beta 1$ homodimers are associated with latency-associated peptide (LAP) non-covalently ${ }^{109}$. The TGF- $\beta 1$-LAP complex is then secreted with chaperon proteins Latent TGF- $\beta$ Binding Proteins (LTBP) by forming the large latent complex (LLC) through disulfide bonds. LLC is anchored to the ECM by LTBP but also connected to cells via integrin recognition of the Arg-Gly-Asp (RGD) motif within the LAP. Immobilization of TGF- $\beta 1$ by the LLC prevents TGF- $\beta 1$ activation and binding to TGF- $\beta$ receptors ${ }^{110}$, providing a repository of latent TGF- $\beta 1$ that can be timely activated in response to various factors. It has been shown that activation of latent TGF- $\beta 1$ is mediated by traction forces exerted by cells on the ECM ${ }^{111}$. Integrin-mediated cell contraction is transmitted to the LAP and induces a conformation change that liberates active TGF- $\beta 1$ (Fig. 1). Importantly, cell-mediated activation of TGF- $\beta 1$ is directly related to the degree of matrix stiffness ${ }^{112}$. Thus, greater amounts of active TGF $\beta 1$ are released on stiff matrices compared with soft ones ${ }^{111}$. During this process, integrins play a major role acting as transmembrane tethers that link the cell cytoskeleton to ECM-bound latent TGF- $\beta 1$. Ultimately, integrins transmit cell generated forces by the actin cytoskeleton to the ECM, which can deform the TGF- $\beta 1$ LLC and release biologically active TGF- $\beta 1$. In the context of lung fibrosis, $\alpha v \beta 6$ integrin expressed in type II 
epithelial cells and $\alpha v \beta 1$ integrin in myofibroblasts have been shown to activate latent TGF- $\beta 1^{113}$. In this regard, $\beta 6$ integrin knockout mice failed to activate latent TGF- $\beta 1$ in vivo and showed protection from lung and kidney fibrosis ${ }^{113-115}$. Accordingly, anti- $\alpha v \beta 6$ integrin blocking antibody (clone 6.3G9) has been shown to prevent TGF- $\beta 1$ activation in vivo and attenuated lung, liver, and kidney fibrosis in mouse models ${ }^{116-118}$. However, a humanized anti- $\alpha v \beta 6$ integrin monoclonal antibody STX-100 from Biogen has been recently stopped in phase $2 b$ trials in patients with IPF due to safety concerns (NCT01371305). Recently, a selective small inhibitor, GSK3008348, is reported to have high affinity to $\alpha v \beta 6$ in human IPF lungs and downregulates pro-fibrotic TGF $\beta$ signaling ${ }^{119}$. Nevertheless, the role of $\alpha v$ integrin in latent TGF- $\beta 1$ activation in fibrotic disease continues to be actively investigated. A fibroblast-specific depletion of $\alpha v$-integrin has been shown to protect mice from lung, kidney, and liver fibrosis, and that inhibition of $\alpha v$ integrins with a pan$\alpha v$ integrin inhibitor (CWHM 12) attenuates fibrosis both in the liver and lungs ${ }^{120}$. Moreover, an anti- $\alpha v$ integrin monoclonal antibody Abituzumab, developed by Merck, has been evaluated in Phase 2 trials in patients with Systemic Sclerosis-associated Interstitial Lung Disease (SSc-ILD); however, the trial was recently terminated due to difficulties in recruiting patients who met the eligibility criteria of the trial (NCT02745145). More recently, a dual selective small molecule inhibitor targeting $\alpha v \beta 6 / \alpha v \beta 1$ integrins PLN-74809, developed by Pliant Therapeutics, is currently being evaluated in Phase 2 a trials in patients with IPF (NCT04072315). Indalo's selective integrin antagonist against $\alpha v \beta 1 / \alpha v \beta 3 / \alpha v \beta 6$, IDL-2965, shows a safe and favorable pharmacokinetics in Phase 1 trials in healthy people and has now entered into multiple-ascending doses trials in patients with IPF (NCT03949530). Other integrin families are also under active research, e.g. $\alpha 6$-integrin is shown to mediate matrix stiffness-regulated myofibroblast invasion and facilitate lung fibrosis ${ }^{121}$.

\section{CONCLUSIONS}

Matrix stiffness remarkedly increases during lung fibrogenesis due to excessive ECM deposition and crosslinking. Traditionally, increased matrix stiffness has been regarded as a consequence of organ fibrosis, however increasing evidence in more recent years has demonstrated that such mechanical factor acts as a major driver of tissue fibrogenesis. Matrix stiffness promotes lung fibrosis through several different means including mechanoactivation of myofibroblasts through integrin-mediated mechanotransduction pathways and activation of the pro-fibrotic cytokine TGF- $\beta 1$. The study of mechanobiology in lung fibrosis is an emerging research field that has prospered with noticeable achievements including the identification of novel therapeutic targets for the treatment of lung fibrosis as well as the development of small molecules and biologics regarded as mechanotherapeutics. This first generation of mechanotherapeutics mainly targets ECM crosslinking enzymes including LOXs/TG2, $\alpha \mathrm{v}$ integrins, mechanosensors such as FAK and ROCK and mechanotransducers including YAP/TAZ or MRTFs; which has been shown to ameliorate organ fibrosis in multiple preclinical models and are currently being investigated in clinical trials in patients with IPF and other fibrotic-related diseases (Table 1). Despite these advancements, it is undoubted that the mechanobiology of 
TABLE 1. Mechanotherapeutics for the treatment of IPF

\begin{tabular}{|c|c|c|c|c|}
\hline Mechanism & Target & Drugs & Company & Status \\
\hline \multirow[t]{4}{*}{ ECM crosslinking } & \multirow[t]{3}{*}{ Lysyl Oxidases } & Simtuzumab (LOXL2 antibody) & Gilead Sciences & Terminated \\
\hline & & PXS-5382A and PXS-5338K (LOXL2 inhibitors) & Pharmaxis & Phase 2 \\
\hline & & PXS-5505 (pan-LOX Inhibitor) & Pharmaxis & Phase 2 for pancreatic cancer \\
\hline & Transglutaminases & ZED 1227 & Zedira & Phase 2 for celiac disease \\
\hline \multirow[t]{4}{*}{ Mechanotransduction } & FAK & Defactinib & Verastem Oncology & Phase 2 for pancreatic cancer \\
\hline & ROCK & KD025 & Kadmon & Phase 2 \\
\hline & MRTFs & CCG-222740, CCG-203971, CCG-1423 & & Preclinical \\
\hline & YAP/TAZ & DRD1 agonists, HMG-CoA reductase inhibitors & & Preclinical \\
\hline \multirow{4}{*}{$\begin{array}{l}\text { Integrin-mediated } \\
\text { TGF- } \beta 1 \text { activation }\end{array}$} & Integrin $\alpha v$ & Abituzumab (anti- $\alpha v$ integrin antibody) & Merck & Terminated \\
\hline & $\alpha v \beta 6$ & STX-100 & Biogen & Terminated \\
\hline & $\alpha v \beta 6 / \alpha v \beta 1$ & PLN-74809 & Pliant & Phase 2 \\
\hline & $\alpha v \beta 1 / \alpha v \beta 3 / \alpha v \beta 6$ & IDL-2965 & Indalo & Phase 1 \\
\hline Epigenetic modulators & miR-21 & RG-012 & Sanofi & Phase 1 for Alport syndrome \\
\hline
\end{tabular}

ECM: extracellular matrix; FAK: focal adhesion kinase; IPF: idiopathic pulmonary fibrosis; LOXL2: lysyl oxidase-like 2; miR: microRNA; MRTF: myocardin-related transcription factor; ROCK: rho-associated protein kinase; TAZ: transcriptional coactivator with PDZ-binding motif; TGF- $\beta 1$ : transforming growth factor $\beta 1$; YAP: yes-associated protein. Adapted from Tschumperlin DJ et al. ${ }^{7}$ with permission from Elsevier, (c) 2020 Elsevier Inc. All rights reserved.

lung fibrosis is far from being fully understood and deserves further investigation in order to unravel the full of spectrum of mechanisms by which biophysical cues control the pathological biology of multiple cell types involved in lung fibrosis. In this regard, a challenging but critical question facing the development of mechanotherapeutics is to target cell-specific mechanisms involved in the development of lung fibrosis without affecting homeostatic functions of healthy cells. Since matrix stiffness is specifically increased during the development of lung fibrosis, activation of pro-fibrotic mechanical signaling pathways is only expected during the development of the disease, thus opening a potential therapeutic window. Together, the identification of cell-specific mechanotransduction pathways involved in lung fibrosis could lead to the development of more selective drugs with a higher therapeutic index, which could represent a real game changer in treatment of lung fibrosis in the future.

\section{ACKNOWLEDGMENTS}

Dr. Lagares gratefully acknowledges funding support from the NIH (grant R01 HL147059-01) and Sponsored Research Grants from Boehringer Ingelheim and Merck \& Co.

\section{DISCLOSURES}

Dr. Lagares declares that he has received research funding from Boehringer Ingelheim, Indalo Therapeutics, and Unity Biotechnology; has a financial interest in Mediar Therapeutics and Zenon Biotech; companies which are 


\section{developing treatments for organ fibrosis. Dr. Lagares, interests were reviewed and are man- aged by MGH and Partners ZealthCare in ac- cordance with their conflicts of interest policies. Dr. Han has no conflicts of interest to report.}

\section{REFERENCES}

1. Wolters PJ, Collard HR, Jones KD. Pathogenesis of idiopathic pulmonary fibrosis. Annu Rev Pathol. 2014;9:157-79.

2. Kendall RT, Feghali-Bostwick CA. Fibroblasts in fibrosis: novel roles and mediators. Front Pharmacol. 2014;5:123.

3. Darby IA, Laverdet B, Bonté F, Desmoulière A. Fibroblasts and myofibroblasts in wound healing. Clin Cosmet Investig Dermatol. 2014;7:301-11.

4. Fasbender F, Widera A, Hengstler JG, Watzl C. Natural Killer Cells and Liver Fibrosis. Front Immun. 2016;7: 19.

5. Hinz B, Lagares D. Evasion of apoptosis by myofibroblasts: a hallmark of fibrotic diseases. Nat Rev Rheumatol. 2020;16:11-31.

6. Phan SH. The myofibroblast in pulmonary fibrosis. Chest, 2002;122(6 Suppl): 286s-9s.

7. Tschumperlin DJ,Lagares D. Mechano-therapeutics: Targeting Mechanical Signaling in Fibrosis and Tumor Stroma. Pharmacol Ther. 2020;212: 107575.

8. Zhou Y, Horowitz JC, Naba A et al. Extracellular matrix in lung development, homeostasis and disease. Matrix Biol. 2018;73:77-104.

9. Hinz B. Mechanical aspects of lung fibrosis: a spotlight on the myofibroblast. Proc Am Thorac Soc. 2012;9:137-47.

10. Lederer DJ, Martinez FJ. Idiopathic Pulmonary Fibrosis. N Engl J Med. 2018;378:1811-23.

11. Barratt SL, Creamer A, Hayton C, Chaudhuri N. Idiopathic Pulmonary Fibrosis (IPF): An Overview. J Clin Med. 2018; 7:201.

12. King TE, Bradford WZ, Castro-Bernardini $S$ et al., A Phase 3 Trial of Pirfenidone in Patients with Idiopathic Pulmonary Fibrosis. N Engl J Med. 2014;370:2083-92.

13. Richeldi L, du Bois RM, Raghu G et al. Efficacy and Safety of Nintedanib in Idiopathic Pulmonary Fibrosis. N Eng J Med. 2014;370: 2071-82.

14. Kato M, Sasaki S, Nakamura $\mathrm{T}$ et al. Gastrointestinal adverse effects of nintedanib and the associated risk factors in patients with idiopathic pulmonary fibrosis. Sci Rep. 2019;9:12062.

15. Mora AL, Rojas M, Pardo A, Selman M. Emerging therapies for idiopathic pulmonary fibrosis, a progressive age-related disease. Nat Rev Drug Disc. 2017;16:755-72

16. Lv M, Liu Y, Ma S, Yu Z., Current advances in idiopathic pulmonary fibrosis: the pathogenesis, therapeutic strategies and candidate molecules. Future Med Chem. 2019;11):2595-2620.

17. Santos A, Lagares D. Matrix Stiffness: the Conductor of Organ Fibrosis. Curr Rheumatol Rep. 2018;20:2.

18. Tschumperlin DJ, Liu F, Tager AM. Biomechanical regulation of mesenchymal cell function. Curr Opin Rheumatol. 2013;25:92-100.

19. Upagupta C, Shimbori C, Alsilmi R, Kolb M. Matrix abnormalities in pulmonary fibrosis. Eur Respir Rev. 2018;27:180033.

20. Booth AJ, Hadley R, Cornett AM et al. Acellular normal and fibrotic human lung matrices as a culture system for in vitro investigation. Am J Respir Crit Care Med. 2012;186:866-76.

21. Liu F, Mih JD, Shea BS et al. Feedback amplification of fibrosis through matrix stiffening and COX-2 suppression. J Cell Biol. 2010;190:693-706.

22. Bonnans C, Chou J, Werb Z. Remodelling the extracellular matrix in development and disease. Nat Rev Mol Cell Biol. 2014;15:786-801.
23. DuFort CC, Paszek MJ, Weaver VM. Balancing forces: architectural control of mechanotransduction. Nat Rev Mol Cell Biol. 2011;12: 308-19.

24. Humphrey JD, Dufresne ER, Schwartz MA. Mechanotransduction and extracellular matrix homeostasis. Nat Rev Mol Cell Biol. 2014;15:802-12.

25. Petersen A, Joly P, Bergmann C, Korus G, Duda GN. The impact of substrate stiffness and mechanical loading on fibroblast-induced scaffold remodeling. Tissue Eng Part A. 2012;18:1804-17.

26. Giménez A, Duch P, Puig M, Gabasa M, Xaubet A, Alcaraz J. Dysregulated Collagen Homeostasis by Matrix Stiffening and TGF- $\beta 1$ in Fibroblasts from Idiopathic Pulmonary Fibrosis Patients: Role of FAK/Akt. Int J Mol Sci.2017;18.

27. Klingberg F, Hinz B, White ES. The myofibroblast matrix: implications for tissue repair and fibrosis. J Pathol. 2013;229:298-309.

28. Hinz B. The myofibroblast: paradigm for a mechanically active cell. J Biomech. 2010;43:146-55.

29. Gabbiani G, Ryan GB, Majne G. Presence of modified fibroblasts in granulation tissue and their possible role in wound contraction. Experientia. 1971;27: 549-50.

30. Hinz B. The role of myofibroblasts in wound healing. Curr Res Transl Med. 2016;64:171-7.

31. El Ghalbzouri A, Hensbergen P, Gibbs S, Kempenaar J, van der Schors R, Ponec M. Fibroblasts facilitate re-epithelialization in wounded human skin equivalents. Lab Invest. 2004;84:102-12.

32. Moulin V, Auger FA, Garrel D, Germain L. Role of wound healing myofibroblasts on re-epithelialization of human skin. Burns. 2000;26:3-12.

33. Freeberg MAT, Perelas A, Rebman JK, Phipps RP, Thatcher TH, Sime PJ Mechanical Feed-Forward Loops Contribute to Idiopathic Pulmonary Fibrosis. Am J Pathol. 2020

34. Bastard C, Bosisio MR, Chabert M et al. Transient micro-elastography: A novel non-invasive approach to measure liver stiffness in mice. World J Gastroenterol. 2011;17:968-75.

35. Lagares D, Ghassemi-Kakroodi P, Tremblay C et al., ADAM10-mediated ephrin-B2 shedding promotes myofibroblast activation and organ fibrosis. Nat Med. 2017;23:1405-15.

36. Espindola MS, Habiel DM, Coelho AL, Mikels-Vigdal A, Hogaboam CM Targeting Lysyl oxidase-like 2 in Idiopathic Pulmonary Fibrosis. BioRxiv. 2019;813907.

37. Oh K, Park HB, Byoun OJ et al. Epithelial transglutaminase 2 is needed for $\mathrm{T}$ cell interleukin-17 production and subsequent pulmonary inflammation and fibrosis in bleomycin-treated mice. J Exp Med. 2011;208:1707-19.

38. Philp CJ, Siebeke I, Clements D et al., Extracellular Matrix Cross-Linking Enhances Fibroblast Growth and Protects against Matrix Proteolysis in Lung Fibrosis. Am J Respir Cell Mol Biol. 2018;58:594-603.

39. Gonzalez-Santamaria J, Villalba M, Busnadiego O et al., Matrix cross-linking lysyl oxidases are induced in response to myocardial infarction and promote cardiac dysfunction. Cardiovasc Res. 2016;109: 67-78.

40. Liu SB, Ikenaga N, Peng ZW et al. Lysyl oxidase activity contributes to collagen stabilization during liver fibrosis progression and limits spontaneous fibrosis reversal in mice. FASEB J. 2016;30:1599-609.

41. Riley DJ, Kerr JS, Berg RA et al. Beta-Aminopropionitrile prevents bleomycin-induced pulmonary fibrosis in the hamster. Am Rev Respir Dis. 1982; 125:67-73.

42. Bellaye PS, Shimbori C, Upagupta C et al., Lysyl Oxidase-Like 1 Protein Deficiency Protects Mice from Adenoviral Transforming Growth Factor- $\beta 1$-induced Pulmonary Fibrosis. Am J Respir Cell Mol Biol. 2018;58:461-70.

43. Raghu G, Brown KK, Collard HR et al. Efficacy of simtuzumab versus placebo in patients with idiopathic pulmonary fibrosis: a randomised, double-blind, controlled, phase 2 trial. Lancet Respir Med. 2017;5:22-32.

44. Puente A, Fortea JI, Cabezas J et al. LOXL2-A New Target in Antifibrogenic Therapy? Int J Mol Sci. 2019;20:1634.

45. Chang J, Lucas MC, Leonte LE et al. Pre-clinical evaluation of small molecule LOXL2 inhibitors in breast cancer. Oncotarget. 2017;8: 26066-78.

46. Hutchinson JH, Rowbottom MW, Lonergan D et al., Small Molecule Lysyl Oxidase-like 2 (LOXL2) Inhibitors: The Identification of an Inhibitor Selective for LOXL2 over LOX. ACS Med Chem Lett. 2017;8:423-7. 
47. Olsen KC, Sapinoro RE, Kottmann RM et al., Transglutaminase 2 and its role in pulmonary fibrosis. Am J Respir Crit Care Med. 2011;184:699-707.

48. Olsen KC, Epa AP, Kulkarni AA et al. Inhibition of transglutaminase 2, a novel target for pulmonary fibrosis, by two small electrophilic molecules. Am J Respir Cell Mol Biol. 2014;50:737-47.

49. Wang K, Zu C, Zhang Y, Wang X, Huan X, Wang L. Blocking TG2 attenuates bleomycin-induced pulmonary fibrosis in mice through inhibiting EMT. Respir Physiol Neurobiol. 2020;276: 103402.

50. Asano S, Ito S, Takahashi K et al. Matrix stiffness regulates migration of human lung fibroblasts. Physiol Rep. 2017;5:e13281.

51. Fiore VF, VWong SS, Tran C et al. $\alpha \mathrm{v} \beta 3$ Integrin drives fibroblast contraction and strain stiffening of soft provisional matrix during progressive fibrosis. JCI Insight. 2018;3: e97597.

52. Lagares D, Santos A, Grasberger PE et al. Targeted apoptosis of myofibroblasts with the BH3 mimetic ABT-263 reverses established fibrosis. Sci Trans1 Med. 2017;9: eaal3765.

53. Jansen KA, Donato DM, Balcioglu HE, Schmidt T, Danen EHJ, Koenderink GH. A guide to mechanobiology: Where biology and physics meet. Biochim Biophys Acta. 2015;1853: 3043-52.

54. Trubelja A, Bao G. Molecular mechanisms of mechanosensing and mechanotransduction in living cells. Extreme Mech Lett. 2018;20:91-8.

55. De R. Cell Mechanosensing. Resonance. 2019;24:289-96.

56. Sun Z, Guo SS, Fässler R. Integrin-mediated mechanotransduction. J Cell Biol. 2016;215:445-6.

57. Jansen KA, Atherton P, Ballestrem C. Mechanotransduction at the cell-matrix interface. Semin Cell Dev Biol. 2017;71:75-83.

58. Fiore VF, Wong SS, Tran $C$ et al. $\alpha v \beta 3$ Integrin drives fibroblast contraction and strain stiffening of soft provisional matrix during progressive fibrosis. JCI Insight. 2018;3:e97597.

59. Patsenker E, Popov Y, Stickel F et al. Pharmacological inhibition of integrin alphavbeta3 aggravates experimental liver fibrosis and suppresses hepatic angiogenesis. Hepatology. 2009;50:1501-11.

60. Reed NI, Jo H, Chen $\mathrm{C}$ et al. The $\alpha v \beta 1$ integrin plays a critical in vivo role in tissue fibrosis. Sci Trans Med. 2015;7:288ra79.

61. Schiller HB, Fassler R. Mechanosensitivity and compositional dynamics of cell-matrix adhesions. EMBO Rep. 2013;14:509-19.

62. Wozniak MA, Modzelewska K, Kwong L, Keely PJ. Focal adhesion regulation of cell behavior. Biochim Biophys Acta. 2004;1692:103-19.

63. Stutchbury B, Atherton P, Tsang R, Wang D-Y, Ballestrem C. Distinct focal adhesion protein modules control different aspects of mechanotransduction. J Cell Sci. 2017;130:1612-24.

64. Moore SW, Roca-Cusachs P, Sheetz MP. Stretchy proteins on stretchy substrates: the important elements of integrin-mediated rigidity sensing. Dev Cell. 2010;19(:194-206.

65. Solon J, Levental I, Sengupta K, Georges PC, Janmey PA. Fibroblast adaptation and stiffness matching to soft elastic substrates. Biophys J. 2007;93:4453-61.

66. Frame MC, Patel H, Serrels B, Lietha D, Eck MJ. The FERM domain: organizing the structure and function of FAK. Nat Rev Mol Cell Biol. 2010;11: 802-14.

67. Lagares D, Busnadiego O, Garcia-Fernandez RA et al., Inhibition of focal adhesion kinase prevents experimental lung fibrosis and myofibroblast formation. Arthritis Rheum. 2012;64:1653-64.

68. Lagares D, Busnadiego O, Garcia-Fernandez RA, Lamas S, Rodriguez-Pascual F. Adenoviral gene transfer of endothelin-1 in the lung induces pulmonary fibrosis through the activation of focal adhesion kinase. Am J Respir Cell Mol Biol. 2012;47:834-42.

69. Jiang H, Hegde S, Knolhoff BL et al. Targeting focal adhesion kinase renders pancreatic cancers responsive to checkpoint immunotherapy. Nat Med. 2016; 22:851-60.

70. Zent J, Guo LW. Signaling Mechanisms of Myofibroblastic Activation: Outside-in and Inside-Out. Cell Physiol Biochem. 2018;49:848-68.

71. Baba I, Egi Y, Utsumi H, Kakimoto T, Suzuki K. Inhibitory effects of fasudil on renal interstitial fibrosis induced by unilateral ureteral obstruction. Mol Med Rep. 2015;12:8010-20.
72. Bei Y, Hua-Huy T, Nicco C et al., RhoA/Rho-kinase activation promotes lung fibrosis in an animal model of systemic sclerosis. Exp Lung Res. 2016; 42:44-55.

73. Jiang C, Huang H, Liu J, Wang Y, Lu Z, Xu Z. Fasudil, a Rho-kinase inhibitor, attenuates bleomycin-induced pulmonary fibrosis in mice. Int J Mol Sci. 2012;13:8293-307.

74. Feng Y, LoGrasso PV, Defert O, Li R. Rho Kinase (ROCK) Inhibitors and Their Therapeutic Potential. J Med Chem. 2016;59:2269-2300.

75. Masumoto A, Hirooka Y, Shimokawa H, Hironaga K, Setoguchi S, Takeshita A. Possible involvement of Rho-kinase in the pathogenesis of hypertension in humans. Hypertension. 2001;38:1307-10.

76. Dupont S, Morsut L, Aragona M et al. Role of YAP/TAZ in mechanotransduction. Nature. 2011;474:179-83.

77. Xu H, Wu X, Qin H et al. Myocardin-Related Transcription Factor A Epigenetically Regulates Renal Fibrosis in Diabetic Nephropathy. J Am Soc Nephrol. 2015;26:1648-60.

78. Seo J, Kim J. Regulation of Hippo signaling by actin remodeling. BMB Rep. 2018;51:151-6.

79. Mohri Z, Del Rio Hernandez A, Krams R. The emerging role of YAP/TAZ in mechanotransduction. J Thorac Dis. 2017;9:E507-9.

80. Reddy P, Deguchi M, Cheng Y, Hsueh AJW. Actin cytoskeleton regulates Hippo signaling. PloS One. 2013;8:e73763.

81. Panciera T, Azzolin L, Cordenonsi M, Piccolo S. Mechanobiology of YAP and TAZ in physiology and disease. Nat Rev Mol Cell Biol. 2017;18:758-70.

82. Noguchi S, Saito A, Mikami Y et al. TAZ contributes to pulmonary fibrosis by activating profibrotic functions of lung fibroblasts. Sci Rep. 2017;7:42595.

83. Szeto SG, Narimatsu M, Lu M et al., YAP/TAZ Are Mechanoregulators of TGF- $\beta$-Smad Signaling and Renal Fibrogenesis. J Am Soc Nephrol. 2016; 27:3117.

84. Liang M, Yu M, Xia R et al., Yap/Taz Deletion in Gli+ Cell-Derived Myofibroblasts Attenuates Fibrosis. J Am Soc Nephrol. 2017;28:3278-90.

85. Toyama T, Looney AP, Baker BM et al. Therapeutic targeting of TAZ and YAP by dimethyl fumarate in systemic sclerosis fibrosis. J Invest Dermatol. 2018;138:78-88.

86. Haak AJ, Kostallari E, Sicard D et al. Selective YAP/TAZ inhibition in fibroblasts via dopamine receptor D1 agonism reverses fibrosis. Sci Transl Med. 2019;11.

87. Santos DM, Pantano L, Pronzati G et al. Screening for YAP Inhibitors Identifies Statins as Modulators of Fibrosis. Am J Respir Cell Mol Biol. 2020;62: 479-92.

88. Finch-Edmondson M, Sudol M. Framework to function: mechanosensitive regulators of gene transcription. Cell Mol Biol Lett. 2016;21:28.

89. Olson EN, Nordheim A. Linking actin dynamics and gene transcription to drive cellular motile functions. Nat Rev Mol Cell Biol. 2010.;11: 353-65.

90. Velasquez LS, Sutherland LB, Liu Z et al. Activation of MRTF-A-dependent gene expression with a small molecule promotes myofibroblast differentiation and wound healing. Proc Natl Acad Sci U S A. 2013;110:16850-5.

91. Shiwen X, Stratton R, Nikitorowicz-Buniak J et al. A Role of Myocardin Related Transcription Factor-A (MRTF-A) in Scleroderma Related Fibrosis. PLoS One. 2015;10:e0126015.

92. Sisson TH, Ajayi IO, Subbotina N et al., Inhibition of myocardin-related transcription factor/serum response factor signaling decreases lung fibrosis and promotes mesenchymal cell apoptosis. Am J Pathol. 2015;185: 969-86.

93. Small EM, Thatcher JE, Sutherland LB et al., Myocardin-related transcription factor-a controls myofibroblast activation and fibrosis in response to myocardial infarction. Circ Res. 2010;107: 294-304.

94. Hutchings KM, Lisabeth EM, Rajeswaran W et al. Pharmacokinetic optimitzation of CCG-203971: Novel inhibitors of the Rho/MRTF/SRF transcriptional pathway as potential antifibrotic therapeutics for systemic scleroderma. Bioorg Med Chem Lett. 2017;27:1744-9.

95. Freeberg MAT, Perelas A, Rebman JK, Phipps RP, Thatcher TH, Sime PJ. Mechanical Feed-Forward Loops Contribute to Idiopathic Pulmonary Fibrosis. Am J Pathol. 2021 Jan;191(1):18-25. 
96. Peng G, Shi X, Kadowaki T. Evolution of TRP channels inferred by their classification in diverse animal species. Mol Phylogenet Evol. 2015;84: 145-57.

97. Peng G, Kashio M, Morimoto T et al., Plant-Derived Tick Repellents Activate the Honey Bee Ectoparasitic Mite TRPA1. Cell Rep. 2015;12:190-202.

98. Nilius B, Owsianik G. The transient receptor potential family of ion channels. Genome Biol. 2011;12:218

99. Peng G, Kashio M, Li T, Dong X, Tominaga M, Kadowaki T. TRPA1 Channels in Drosophila and Honey Bee Ectoparasitic Mites Share Heat Sensitivity and Temperature-Related Physiological Functions. Front Physiol. 2016; 7:447.

100. Rahaman SO, Grove LM, Paruchuri S et al. TRPV4 mediates myofibroblast differentiation and pulmonary fibrosis in mice. JCI. 2014;124:5225-38.

101. Adapala RK, Thoppil RJ, Luther DJ et al. TRPV4 channels mediate cardiac fibroblast differentiation by integrating mechanical and soluble signals. J Mol Cell Cardiol. 2013;54:45-52.

102. Beech David J, Kalli Antreas C. Force Sensing by Piezo Channels in Cardiovascular Health and Disease. Arterioscler Thromb Vasc Biol. 2019;39: 2228-39.

103. Beech DJ, Xiao B. Piezo channel mechanisms in health and disease. J Physiol. 2018;596:965-67.

104. Aykut B, Chen R, Kim JI et al., Targeting Piezo1 unleashes innate immunity against cancer and infectious disease. Sci Immun. 2020;5:eabb5168.

105. Blythe NM, Muraki K, Ludlow MJ et al., Mechanically activated Piezo1 channels of cardiac fibroblasts stimulate p38 mitogen-activated protein kinase activity and interleukin-6 secretion. J Biol Chem. 2019;294: 17395-408.

106. Branton MH, Kopp JB. TGF-beta and fibrosis. Microbes Infect. 1999;1: 1349-65.

107. Varga J, Pasche B. Transforming growth factor beta as a therapeutic target in systemic sclerosis. Nat Rev Rheumatol. 2009;5:200-6.

108. Sanjabi S, Oh SA, Li MO. Regulation of the Immune Response by TGF- $\beta$ : From Conception to Autoimmunity and Infection. Cold Spring Harbor perspectives in biology, 2017. 9(6): p. a022236.
109. Munger, J.S. and D. Sheppard, Cross talk among TGF-beta signaling pathways, integrins, and the extracellular matrix. Cold Spring Harb Perspect Biol. 2011;3:a005017.

110. Robertson IB, Rifkin DB. Regulation of the Bioavailability of TGF- $\beta$ and TGF- $\beta$-Related Proteins. Cold Spring Harb Perspect Biol. 2016;8:a021907.

111. Klingberg F, Chow ML, Koehler A et al. Prestress in the extracellular matrix sensitizes latent TGF- $\beta 1$ for activation. J Cell Biol. 2014;207:283-97.

112. Hinz B. The extracellular matrix and transforming growth factor- $\beta 1$ : Tale of a strained relationship. Matrix Biol. 2015;47:54-65.

113. Munger JS, Huang X, Kawakatsu $\mathrm{H}$ et al. The integrin alpha v beta 6 binds and activates latent TGF beta 1: a mechanism for regulating pulmonary inflammation and fibrosis. Cell. 1999;96:319-28.

114. Ma LJ, Yang H, Gaspert A et al., Transforming growth factor-beta-dependent and -independent pathways of induction of tubulointerstitial fibrosis in beta6(-/-) mice. Am J Pathol. 2003;163:1261-73.

115. Hahm K, Lukashev ME, Luo Y et al. Alphav beta6 integrin regulates renal fibrosis and inflammation in Alport mouse. Am J Pathol. 2007;170:110-25.

116. Horan GS, Wood S, Ona V et al. Partial inhibition of integrin alpha(v) beta6 prevents pulmonary fibrosis without exacerbating inflammation. Am J Respir Crit Care Med. 2008;177:56-65.

117. Wang B, Dolinski BM, Kikuchi N et al., Role of alphavbeta6 integrin in acute biliary fibrosis. Hepatology. 2007;46:1404-12.

118. Peng ZW, Ikenaga N, Liu SB et al. Integrin $\alpha v \beta 6$ critically regulates hepatic progenitor cell function and promotes ductular reaction, fibrosis, and tumorigenesis. Hepatology. 2016;63:217-32.

119. John AE, Graves RH, Pun KT et al., Translational pharmacology of an inhaled small molecule $\alpha v \beta 6$ integrin inhibitor for idiopathic pulmonary fibrosis. Nat Commun. 2020;11:4659.

120. Henderson NC, Arnold TD, Katamura Y et al., Targeting of alphav integrin identifies a core molecular pathway that regulates fibrosis in several organs. Nat Med. 2013;19:1617-24

121. Chen $\mathrm{H}, \mathrm{Qu}$ J, Huang $X$ et al., Mechanosensing by the $\alpha 6$-integrin confers an invasive fibroblast phenotype and mediates lung fibrosis. Nat Commun 2016;7:12564. 